\title{
Wave analysis of porous geometry with linear resistance law
}

\author{
Jørgen Dokken, John Grue* and Lars Petter Karstensen
}

Mechanics Division, Department of Mathematics, University of Oslo, 0316 Oslo, Norway

\begin{abstract}
The wave diffraction-radiation problem of a porous geometry of arbitrary shape located in the free surface of a fluid is formulated by a set of integral equations, assuming a linear resistance law at the geometry. The linear forces, the energy relation and the mean horizontal drift force are evaluated for non-porous and porous geometries. A geometry of large porosity has an almost vanishing added mass. The exciting forces are a factor of 5-20 smaller compared to a solid geometry. In the long wave regime, the porous geometry significantly enhances both the damping and the mean drift force, where the latter grows linearly with the wavenumber. The calculated mean drift force on a porous hemisphere and a vertical truncated cylinder, relevant to the construction of fish cages, is compared to available published results.
\end{abstract}

Keywords: Wave analysis, fish cages, mean drift force, wave exciting force, added mass, damping

Article ID: 1671-9433(2017)01-0020-07

\section{Introduction $^{1}$}

Analysis of the loads on floating bodies is an established research discipline (see Faltinsen, 1990) including wave-current-body interaction (see Grue and Palm, 1996) and computational advances of complex geometries (see Newman, 2014). Besides the more common wave analysis of solid floating bodies, more general geometries with slots or pores have caught industrial interest, where one of the applications has been to enhance the damping and reduce the wave loads on offshore structures (see Molin, 2011). Another application is tuned liquid damping of the lateral motions of

\footnotetext{
${ }^{1}$ Received date: dd-mm-yyyy

Accepted date: dd-mm-yyyy

*Corresponding author Email:johng@math.uio.no

(C) Harbin Engineering University and Springer-Verlag Gmbh Germany 2017
}

land buildings (see Molin and Remy, 2013). The aerodynamic effect of a porous geometry and flow through screens have been analyzed (see Taylor, 1956; Laws and Livesey, 1978). It is commonly assumed that the geometry is represented by a thin wall with the effect of the screens or fine pores modelled by a relation between the pressure jump $(\Delta p)$ and normal velocity $\left(w_{n}\right)$, where a linear or quadratic resistance law is assumed.

Wave interaction with porous geometries assuming a pressure jump that is linear in $w_{n}$ has been investigated by Chwang (1983), Chwang and Wu (1994), Chwang and Chan (1998) and Williams et al. (2000). In relation to coastal engineering purposes and the practical construction of breakwaters $\mathrm{Yu}$ (1995) demonstrated that the neglect of the inertia effect underestimates the functional performance of such porous structures. Huang et al. (2011) reviewed the transmission and reflection characteristics and the hydraulic performance of perforated coastal breakwaters including Jarlan (1961) type geometries with or without a back-wall. Wave reflection and transmission from breakwaters were further studied by Liu and Li (2013) while Koley et al. (2015a, 2015b) modelled oblique wave scattering and wave trapping at a vertical flexible porous plate, including also a two-layer fluid, see Behera et al. (2015).

In relation to marine and offshore hydrodynamics, Zhao et al. (2011a) developed generalizations of the common expressions of the linear radiation and diffraction forces as well as obtaining the mean drift force, where calculations were obtained for a vertical truncated cylinder assuming normal mode expansions of the external and internal wave potentials. In Zhao et al. (2011b) they obtained experimental relations between the porosity factor and the opening fraction of the geometry, in combination with the wave slope of the incoming waves. A quadratic relation between the pressure drop and normal velocity, i.e., $\Delta p=$ const. $\times w_{n}^{2}$, has been pursued by Molin (2001, 
2011), An and Faltinsen (2012) and Molin and Remy (2013), in wave radiation and diffraction studies of porous or slotted geometries.

In the present paper, the main novelties, for a porous geometry located in the free surface, assuming a linear resistance law, include: a) a wave analysis of porous geometries of arbitrary shape; b) a formulation based on integral equations valid for bodies in three dimensions; c) numerical representation and calculation of the various force coefficients of the general body including the energy balance; d) particular efforts obtaining the linear exciting force and mean drift force where the latter is second order in the wave amplitude; e) practical evaluation of these quantities, where the exciting force and mean drift force are important quantities in a wave analysis of floating fish farms, for example. The evaluation of the mean drift force on the porous geometry - of arbitrary shape - is a particular novel contribution, since none of the above mentioned publications have considered this force, or its numerical values have been obtained erroneously as in Zhao et al. (2011a). Thus, particularly, section 5 of the paper is devoted to the evaluation of the mean drift force. Computations for porous and solid geometries including a hemisphere and a truncated vertical cylinder where both geometries are relevant for the case of fish cages - are carried out with comparison to existing publications of the solid geometry case published by Grue and Biberg (1993) and Molin (1994).

The paper is organized as follows: Section 2 describes the mathematical representation of the flow exterior and interior to the geometry exposed to the incoming waves as well as the radiation problem. In section 3 we develop the kinematic-dynamic boundary condition at the porous geometry. Section 4 describes the linear forces including added mass, damping and the exciting forces, as well as the energy relation, and their numerical evaluation. In section 5 we rederive the mathematical expression for the mean horizontal drift force and perform numerical calculations for solid and porous geometries with comparison to existing publications for the solid body case (Grue and Biberg, 1993; Molin, 1994) as well as for the porous body case (Zhao et al., 2011a) correcting the numerical calculations in the latter for an obvious error. Finally section 6 is a conclusion.

\section{Mathematical formulation}

A coordinate system is introduced with the $x$ and $y$ axis in the horizontal plane and $z$ the vertical axis, with the water surface at rest at $z=0$ (Fig. 1). The incoming waves interact with a floating geometry which is assumed to have a porous boundary. The geometry is floating because of a buoyant element at the intersection between the geometry and the free surface, but this part of the geometry is disregarded in the present analysis where only the effect of the main porous geometry is considered. The fluid motion exterior and interior to the geometry is assumed to be inviscid and irrotational, and obtained by linear potential theory. The analysis is carried out in the frequency domain where the (periodic) incoming waves are characterized by the frequency $\omega$, wavenumber $K$, wave angle $\beta$ and wave amplitude $A$.

\subsection{Representation of the exterior and interior wave fields}

We assume in this application that the motion of the porous geometry may be modelled as a rigid body motion. The more general case where the geometry is flexible may be modelled within linear theory, assuming an additional set of motion modes corresponding to the flexible modes of the geometry. The flexible case is left for future study.

A periodic motion in time of frequency $\omega$ is assumed. Assuming a rigid body motion, the velocity at a position $P$ on the geometry, with coordinates $\left(x_{P}, y_{P}, z_{P}\right)$, is given by $\mathbf{v}_{P}=$ $\left(v_{1}, v_{2}, v_{3}\right)_{P}=\operatorname{Re}\left\{e^{\mathrm{i} \omega t} \mathrm{i} \omega\left[\left(\xi_{1}, \xi_{2}, \xi_{3}\right)+\left(\xi_{4}, \xi_{5}, \xi_{6}\right) \times\right.\right.$ $\left.\left.\left(x_{P}, y_{P}, z_{P}\right)\right]\right\}$, where the motion amplitudes $\xi_{j}$ of the six degrees of freedom correspond to the translatory modes in surge, sway and heave $(j=1,2,3)$, respectively, and the rotational modes in roll, pitch and heave, about the $x, y$ and $z$ axes $(j=4,5,6)$, respectively.

The wave potentials describing the fluid motion in the exterior and interior domains (see Fig. 1), $\tilde{\phi}^{E}$ and $\tilde{\phi}^{I}$, respectively, may be expressed by

$$
\begin{aligned}
& \tilde{\phi}^{E, I}(x, y, z, t)=\tilde{\phi}_{D}^{E, I}(x, y, z, t)+\tilde{\phi}_{\text {rad }}^{E, I}(x, y, z, t) \\
& =\operatorname{Re}\left(e^{\mathrm{i} \omega t}\left[\frac{\mathrm{i} A g}{\omega} \phi_{D}^{E, I}(x, y, z)+\mathrm{i} \omega \sum_{j=1}^{6} \xi_{j} \phi_{j}^{E, I}(x, y, z)\right]\right),
\end{aligned}
$$

where $\phi_{D}^{E, I}$ and $\phi_{j}^{E, I}$ denote the diffraction potential and radiation potential of motion mode number $j$, respectively, in the exterior and interior domains, and $g$ denotes the acceleration of gravity (and $A$ is the wave amplitude). The diffraction potentials $\phi_{D}^{E, I}$ are composed by the velocity potential $\phi_{0}$ due to the incoming waves plus the remainders $\phi_{7}^{E, I}=\phi_{D}^{E, I}-\phi_{0}$.

Assuming deep water, the incoming wave potential is given by

$$
\phi_{0}=e^{K z-\mathrm{i} K(x \cos \beta+y \sin \beta)},
$$


where $\beta$ denotes the wave angle, with $\beta=0$ corresponding to incoming waves propagating along the positive $x$-axis.

The velocity potentials $\phi_{j}^{E, I}(j=1, \ldots, D)$ satisfy the Laplace equation in the respective fluid domains. The linear free surface boundary condition applies at $z=0$ :

$$
-K \phi_{j}^{E, I}+\frac{\partial \phi_{j}^{E, I}}{\partial z}=0, \quad j=1, \ldots, D
$$

where $K=\omega^{2} / g$. Further, the potentials $\phi_{j}^{E}$ in the exterior domain satisfy a radiation condition in the far field:

$$
\frac{\partial \phi_{j}^{E}}{\partial R}=-\mathrm{i} K \phi_{j}^{E}+O\left(\frac{1}{R}\right), R \rightarrow \infty, j=1, \ldots, 7,
$$

where $R=\sqrt{x^{2}+y^{2}}$. The velocities $\left|\nabla \phi_{j}^{E}\right| \rightarrow 0$ for $z \rightarrow-\infty$. A remaining boundary condition relating the normal velocity to the pressure drop at the geometry, assuming a linear relationship is obtained in section 3 below.

The potentials $\phi_{j}^{E, I}(j=1, \ldots, D)$ are obtained by a set of integral equations. The exterior potentials $\phi_{j}^{E}$ are obtained by, for a point $\left(x^{\prime}, y^{\prime}, z^{\prime}\right)$ on the porous geometry:

$$
2 \pi \phi_{j}^{E^{\prime}}+\int_{S_{B}}\left(\phi_{j}^{E} \frac{\partial G}{\partial n}-G \frac{\partial \phi_{j}^{E}}{\partial n}\right) d S=4 \pi \phi_{0}^{\prime} \delta_{D, j}
$$

$(j=1, \ldots, D)$, and for a point $\left(x^{\prime}, y^{\prime}, z^{\prime}\right)$ in the exterior fluid:

$$
4 \pi \phi_{j}^{E^{\prime}}+\int_{S_{B}}\left(\phi_{j}^{E} \frac{\partial G}{\partial n}-G \frac{\partial \phi_{j}^{E}}{\partial n}\right) d S=4 \pi \phi_{0}^{\prime} \delta_{D, j},
$$

$(j=1, \ldots, D$.$) Here, G$ denotes the wave Green function corresponding to a rankine source in the point $(x, y, z)$, satisfying the linear free surface boundary condition at $z=0$, the radiation condition in the far field as well as the bottom boundary condition for $z \rightarrow-\infty$. The function $G$ is specified in section 2.2 below. Further, $\delta_{D, j}$ on the r.h.s. of (5) denotes the Kroenecker-delta, a prime means evaluation at the point $\left(x^{\prime}, y^{\prime}, z^{\prime}\right)$ and the normal $n$ points out of the exterior fluid. The internal potentials are obtained similarly, for a point $\left(x^{\prime}, y^{\prime}, z^{\prime}\right)$ on the porous geometry,

$$
-2 \pi \phi_{j}^{I^{\prime}}+\int_{S_{B}}\left(\phi_{j}^{I} \frac{\partial G}{\partial n}-G \frac{\partial \phi_{j}^{I}}{\partial n}\right) d S=0,
$$

$(j=1, \ldots, D)$, where a minus appears in front of the first term on the 1.h.s. of (7) since the normal $n$ is pointing into the interior domain.
Sum and difference potentials along the geometry are introduced by $\Phi_{j}=\phi_{j}^{E}+\phi_{j}^{I}$ and $\Psi_{j}=\phi_{j}^{E}-\phi_{j}^{I}$ $(j=1, \ldots, D)$. Combination of (5) and (7) gives:

$$
\begin{gathered}
2 \pi \Phi_{j}^{\prime}+\int_{S_{B}} \Psi_{j} \frac{\partial G}{\partial n} d S=4 \pi \phi_{0}^{\prime} \delta_{D, j}, \\
2 \pi \Psi_{j}^{\prime}+\int_{S_{B}} \Phi_{j} \frac{\partial G}{\partial n} d S=\int_{S_{B}} G \frac{\partial \Phi_{j}}{\partial n} d S+4 \pi \phi_{0}^{\prime} \delta_{D, j},
\end{gathered}
$$

$(j=1, \ldots, D)$, where in $(8)$ we have used that the normal velocity is continuous at the boundary, see also (19) below. (a)

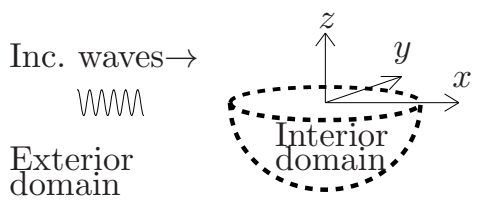

(b)

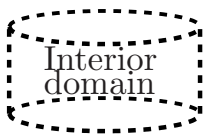

Fig. 1 Coordinate system and sketch of (a) porous hemisphere and (b) truncated vertical cylinder.

\subsection{Far field behavior of the Green function and the potentials}

The Green function is given by

$$
G=\frac{1}{r}+\int_{0}^{\infty} \frac{k+K}{k-K} e^{k\left(z+z^{\prime}\right)} J_{0}\left(k r_{0}\right) d k
$$

where $r=\sqrt{\left(x-x^{\prime}\right)^{2}+\left(y-y^{\prime}\right)^{2}+\left(z-z^{\prime}\right)^{2}} \mid, \quad r_{0}=$ $\sqrt{\left(x-x^{\prime}\right)^{2}+\left(y-y^{\prime}\right)^{2}}, J_{0}$ denotes the Bessel function of first kind and order zero, and the path of integration is above the pole at $k=K$. For $R^{\prime}=\sqrt{x^{\prime 2}+y^{\prime 2}} \rightarrow \infty$, $G$ takes the form, see Grue and Palm (1993, eq. (4.1)),

$$
G=R^{\prime-1 / 2} h\left(\theta^{\prime}\right) e^{K z^{\prime}-\mathrm{i} K R^{\prime}}\left(1+O\left(\left(K R^{\prime}\right)^{-1}\right)\right)
$$

where the angle $\theta^{\prime}$ is related to the far field coordinates by $\left(x^{\prime}, y^{\prime}\right)=R^{\prime}\left(\cos \theta^{\prime}, \sin \theta^{\prime}\right)$ and

$$
h\left(\theta^{\prime}\right)=(8 \pi K)^{1 / 2} e^{K\left(z+\mathrm{i} x \cos \theta^{\prime}+\mathrm{i} y \sin \theta^{\prime}\right)-\mathrm{i} \pi / 4} .
$$

The potentials are obtained in the far field by (6)

$$
\begin{aligned}
& \phi_{j}^{E^{\prime}}\left(x^{\prime}, y^{\prime}, z^{\prime}\right)=-\frac{1}{4 \pi} \int_{S_{B}}\left(\phi_{j}^{E} \frac{\partial G}{\partial n}-G \frac{\partial \phi_{j}^{E}}{\partial n}\right) d S \\
& =\left(\frac{K}{2 \pi R}\right)^{1 / 2} H_{j}\left(\theta^{\prime}\right) e^{K\left(z^{\prime}-\mathrm{i} R^{\prime}\right)}+O\left(\frac{1}{R}\right),
\end{aligned}
$$

$(j=1, \ldots, 7)$, where

$H_{j}=\int_{S_{B}}\left(\frac{\partial \phi_{j}^{E}}{\partial n}-\phi_{j}^{E} \frac{\partial}{\partial n}\right) e^{K\left(z+\mathrm{i} x \cos \theta^{\prime}+\mathrm{i} y \cos \theta^{\prime}\right)-\mathrm{i} \pi / 4} d S$, 


$$
\begin{aligned}
(j & =1, \ldots, 6) \\
H_{7} & =\int_{S_{B}}\left(\frac{\partial \phi_{D}^{E}}{\partial n}-\phi_{D}^{E} \frac{\partial}{\partial n}\right) e^{K\left(z+\mathrm{i} x \cos \theta^{\prime}+\mathrm{i} y \cos \theta^{\prime}\right)-\mathrm{i} \pi / 4} d S .
\end{aligned}
$$

\section{Kinematic-dynamic boundary condition at the geometry}

The wave induced motion sets up a local flow through the porous geometry. Let $w_{n}=\left(\mathbf{v}^{E, I}-\mathbf{v}_{P}\right) \cdot \mathbf{n}$ denote the velocity normal to the geometry where $\mathbf{v}^{E}=\nabla \tilde{\phi}^{E}$ and $\mathbf{v}^{I}=\nabla \tilde{\phi}^{I}$ denote the velocity of the exterior and interior fluid, respectively, and $\mathbf{v}_{P}$ is defined in the text above (1). A resistance law at the geometry is generally of the form $w_{n}=F(\Delta p)$ where $\Delta p$ denotes the pressure drop between the exterior and interior fluid. Following Taylor (1956) studying aerodynamical flow applications $\Delta p=\left(\rho C_{0} / 2\right) w_{n}^{2}$ models a quadratic resistance law and $\Delta p=(\mu / b) w_{n}$ a linear law where $\rho$ denotes density, $C_{0}$ a dimensionless coefficient, $\mu$ viscosity and $b$ a coefficient with dimension. Here we pursue the linear pressure drop assumption which is a valid approach for geometries with fine pores, where

$$
w_{n}=\frac{b}{\mu} \Delta p
$$

at $S_{B}$. The ratio $\rho b / \mu$ has dimension of time over length.

Wave generation by a moving porous plate assuming a linear pressure drop was studied by Chwang (1983) and Chwang and $\mathrm{Li}$ (1983), expressing the porosity effect by a parameter $G_{0}=\rho b \omega / K \mu$ where $K=\omega^{2} / g$. Zhao et al. (2011a) studied the linear and mean drift forces on a vertical porous circular cylinder as function of the parameter ' $b^{\prime}=B_{0}=2 \pi G_{0}$ and the wavenumber. By a set of experiments Zhao et $a l$. (2011b) connected the porosity parameter $B_{0}$ to the opening rate $\tau$ of the material as well as the waveslope $\epsilon=K A$, see particularly their equation (29), obtaining

$$
B_{0}=\frac{(17.8 / \epsilon+143.2) \tau^{2}}{1+1.06 \tau} .
$$

Table 1 illustrates practical values of $\tau$ as function of $B_{0}=2 \pi G_{0}$ obtained from (17) where a waveslope of $K A=0.05$ is assumed.

Returning to the derivation of a boundary condition at the geometry we obtain the pressure drop by $\Delta p=p^{E}-p^{I}$, where $p^{E}, p^{I}$ denote the pressure of the exterior and interior fluid, respectively. The Bernoulli equation gives, assuming a linear motion: $\Delta p=-\rho\left(\partial \tilde{\phi}^{E} / \partial t-\partial \tilde{\phi}^{I} / \partial t\right)$. Using $w_{n}=(b / \mu) \Delta p$
Table 1 Porosity parameter $B_{0}$ and the corresponding opening rate $\tau$ of the geometry for $K A=0.05$ using (17).

\begin{tabular}{cccccc}
\hline$B_{0}$ & $2 \pi$ & 8.5 & $5 \pi$ & $10 \pi$ & $20 \pi$ \\
\hline$\tau$ & 0.12 & 0.14 & 0.19 & 0.29 & 0.43 \\
\hline
\end{tabular}

we obtain

$$
\begin{aligned}
& -\frac{\mathrm{i} \rho b \omega}{\mu}\left(\frac{\mathrm{i} A g}{\omega} \Psi_{D}+\mathrm{i} \omega \sum_{j=1}^{6} \xi_{j} \Psi_{j}\right) \\
& =\frac{\mathrm{i} A g}{\omega} \frac{\partial \phi_{D}^{E, I}}{\partial n}+\mathrm{i} \omega \sum_{j=1}^{6} \xi_{j}\left(\frac{\partial \phi_{j}^{E, I}}{\partial n}-n_{j}\right),
\end{aligned}
$$

giving

$$
\begin{aligned}
\frac{\partial \phi_{j}^{E}}{\partial n} & =\frac{\partial \phi_{j}^{I}}{\partial n}=n_{j} \delta_{j J}-\frac{\mathrm{i} \rho b \omega}{\mu}\left(\phi^{E}-\phi^{I}\right)_{j}, \\
\frac{\partial \Phi_{j}}{\partial n} & =2 n_{j} \delta_{j J}-\frac{2 \mathrm{i} \rho b \omega}{\mu} \Psi_{j}
\end{aligned}
$$

$(j=1, \ldots, D, J=1, \ldots, 6)$, in both equations, where the relations close the set of equations (8-9) for the potentials $\Phi_{j}, \Psi_{j}$ as well as $\phi_{j}^{E}, \phi_{j}^{I}$.

Numerical solution of the set of integral equations $(8-9,19-20)$ is obtained by two different codes. One is a Python-script derived specifically for the unbounded fluid case where the Green function is replaced by the Rankine source $\left(G=1 /\left|(x, y, z)-\left(x^{\prime}, y^{\prime}, z^{\prime}\right)\right|\right)$ and the fluxes are evaluated by high-order Gauss quadrature. The other is a diffraction-radiation panel code which is a generalization of the low-order version of the WAMIT-code (wamit.inc), see also Nossen et al. (1991), Grue and Palm (1996) and Finne and Grue (1997). Geometries explored in the present paper include a sphere in unbounded fluid and a hemisphere as well as a truncated vertical cylinder in the free surface. The number of panels is $N=1024$ or 2048 for the sphere, $N=1024$ for the hemisphere and $N=1131$ for the truncated cylinder. Running two different codes for the case of the submerged sphere makes a double check of the computations of the effect of porosity at the geometry, without the wave effects. The calculations involving the wave effects with the geometries in the free surface are qualified by evaluation of energy checks and obtaining the linear exciting force in two different ways. In the calculations of the mean drift force in section 5 we compare with published results for the case of a solid hemisphere and a truncated cylinder at finite and infinite water depth published in Grue and Biberg (1993) as well as in Molin (1994). 

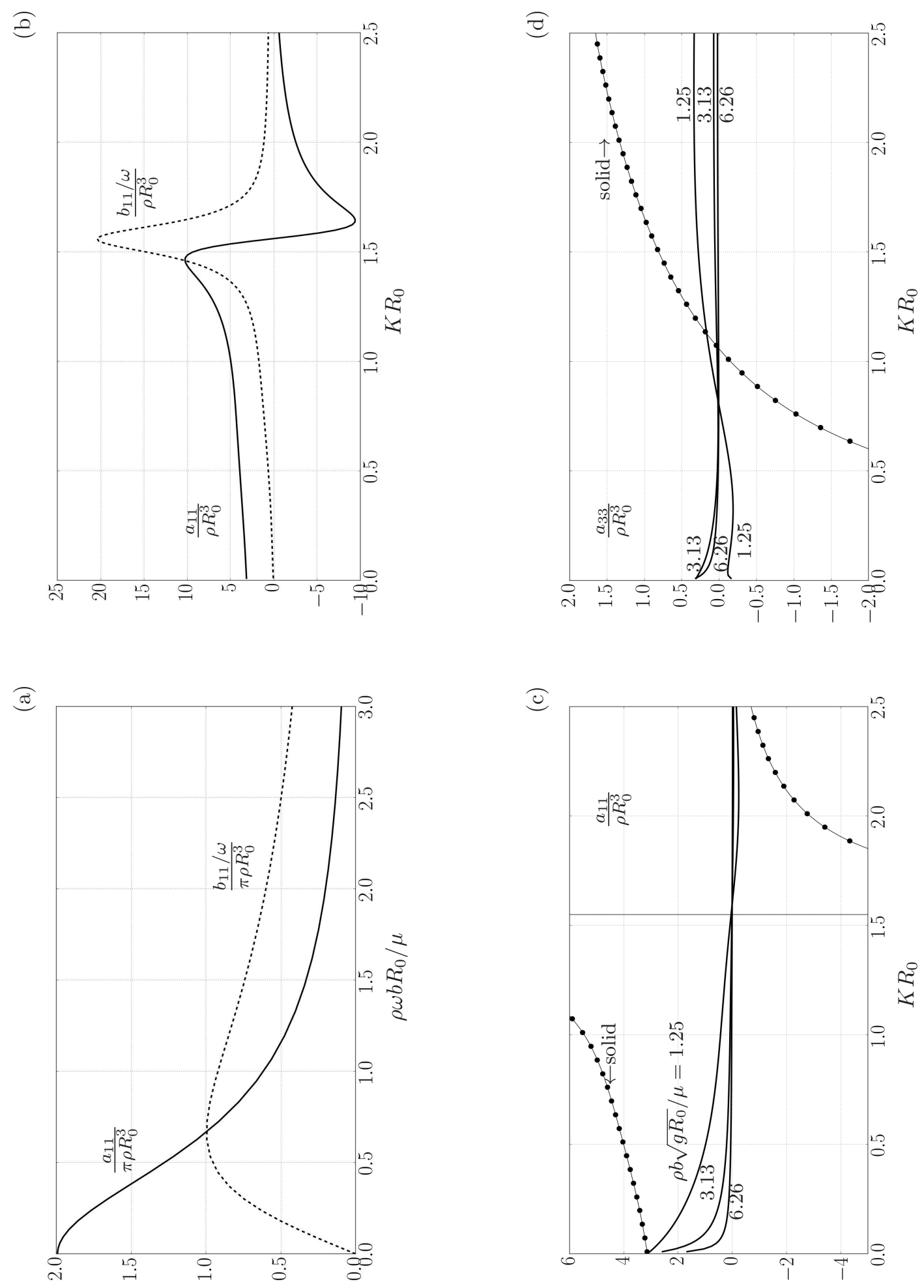

Fig. 2 Added mass and damping coefficients $a_{11}$ and $b_{11}$. (a) Porous sphere of radius $R_{0}$ in unbounded fluid.

(b) Floating hemisphere of radius $R_{0}$ for $\rho b \sqrt{g R_{0}} / \mu=0.157$. (c) Same as b) but $a_{11}$ for $\rho b \sqrt{g R_{0}} / \mu=1.25,3.13$, 6.26. (d) Same as (c) but $a_{33}$. 


\section{Linear forces}

A set of relations of the linear force coefficients have by Zhao et al. (2011a) been obtained by use of Green's theorem to the set of wave potentials. The relations are generalizations of the usual added mass and damping symmetry and the Haskind-relation in the diffraction problem for solid geometries, where a linear resistance law is assumed for the porous case. We here evaluate the added mass and damping force which in unbounded fluid are functions of the parameter $\rho \omega b R_{0} / \mu$. A main effect of the porous geometry is to introduce a significant damping. The coupling between the exterior and interior motion further modifies the forces. In the free surface case the contributions to the damping coefficients from the dissipation at the geometry and the radiated waves are obtained. The exciting force is calculated by both pressure integration and the generalized Haskind-relations which are rederived here. In the diffraction problem the incoming and scattered energy flux balances exactly the porous energy loss.

\subsection{Added mass and damping}

The forces and moments in the radiation problem are obtained by integrating the linear pressure over the body surface including the outer and inner motion, giving, $F_{i}(t)=\operatorname{Re}\left(\xi_{j}\left(\omega^{2} a_{i j}-\mathrm{i} \omega b_{i j}\right) e^{\mathrm{i} \omega t}\right)$, where $a_{i j}$ and $b_{i j}$ denote the added mass and damping coefficients, respectively, obtained by:

$$
a_{i j}-\mathrm{i} b_{i j} / \omega=\rho \int_{S_{B}} \Psi_{j} n_{i} d S, \quad i, j=1,2, \ldots 6 .
$$

The symmetry of $a_{i j}$ and $b_{i j}$ was demonstrated by Zhao et al. (2011a).

The work per unit time performed by the force opposing the pressure force on the body, in the radiation mode $J$, averaged in time, is expressed by

$$
\overline{W_{J}}=-\overline{\int_{S_{B}}\left(p_{r a d, J}^{E}-p_{r a d, J}^{I}\right) \mathbf{n} \cdot \mathbf{v}_{P, J} d S},
$$

where $p_{\text {rad,J }}^{E, I}$ denotes the external/internal pressure in the radiation mode number $J, \mathbf{v}_{P, J}$ the velocity at the point $P$ on the geometry $S_{B}$ for mode $J$ and an overline time average. Carrying out the time average in (22) we obtain $\overline{W_{J}}=-(1 / 2) \rho \omega^{3}\left|\xi_{J}\right|^{2} \operatorname{Im} \int_{S_{B}} \Psi_{J} n_{J} d S=$ $\omega^{2}\left|\xi_{J}\right|^{2} b_{J J} / 2$.

The work in (22) contributes to a) an energy loss due to the pressure drop across the porous geometry, given by $\int_{S_{B}} \Delta p w_{n} d S$, and b) an energy flux at a control surface $S_{\infty}$ in the far field given by $\overline{\int_{S_{\infty}}-\rho\left(\partial \phi_{\text {rad }, J}^{E} / \partial t\right)\left(\partial \phi_{\text {rad }, J}^{E} / \partial n\right) d S}$ where $\mathbf{n}$ is point- ing out of the external fluid. By carrying out the respective integrals we obtain

$$
\frac{b_{J J}}{\rho \omega}=\frac{b \omega}{\mu} \int_{S_{B}}\left|\Psi_{J}\right|^{2} d S+\frac{K}{4 \pi} \int_{0}^{2 \pi}\left|H_{J}(\theta)\right|^{2} d \theta,
$$

where the first term expresses the dissipation along the porous geometry and in the latter integral the far field form (13) has been used with $H_{J}$ obtained in (14). We note that (23) is here derived directly from the energy balance equation, a superior principle. Using a different method and Green's theorem, Zhao et al. (2011a, eq. (17)) have derived a set of relations for the damping coefficients $b_{i j}$ but these are not used in the present calculations.

Fig. 2(a) illustrates the added mass and damping coefficients $a_{11}$ and $b_{11} / \omega$ for a sphere of radius $R_{0}$ in unbounded fluid as function of the parameter $\rho \omega b R_{0} / \mu$. In the case when $\rho \omega b R_{0} / \mu \rightarrow 0$ the added mass coefficient tends to $a_{11} \rightarrow 2 \pi \rho R_{0}^{3}$ where a contribution of $(2 / 3) \pi \rho R_{0}^{3}$ corresponds to the added mass due to the external flow and another contribution of $(4 / 3) \pi \rho R_{0}^{3}$ is due to the internal flow, where the latter is just the displaced volume of the sphere. The two different codes produce the same results (results not shown).

Fig. 2(b) shows the added mass and damping coefficients of a floating hemisphere of radius $R_{0}$, of small porosity with $\rho b \sqrt{g R_{0}} / \mu=0.094$. The forces, function of the wavenumber $K R_{0}$, exhibit a high, finite damping coefficient close to the resonance at $K R_{0}=\pi / 2$. A finite added mass is observed close to the resonance frequency as well. The added mass is negative for the computed frequencies with $K R_{0}>\pi / 2$. The added mass coefficients $a_{11}$ and $a_{33}$ are approximately zero for the higher porosity factors with $\rho b \sqrt{g R_{0}} / \mu$ up to 6.26 for all $K R_{0}$ (Figs. 3(c,d)).

The floating hemisphere with a small porosity factor of $\rho b \sqrt{g R_{0}} / \mu=0.157$ exhibits a large porous damping in vicinity of the resonance frequency, in the lateral mode of motion $\left(b_{11}\right)$. The wave radiation contribution is comparatively small (Fig. 3(a)). The dissipation of the porous geometry is high in the long wave range in the heave mode of motion $\left(b_{33}\right)$ (Fig. $3(\mathrm{~b})$ ). The hemisphere with larger porosity factors $\rho b \sqrt{g R_{0}} / \mu$ up to 12.53 exhibits a dissipation that is always exceeding the damping of the solid geometry, in the long wave range, in both sway and heave (Figs. 3(c,d)).

Note that the calculations of $b_{j j}$ using the pressure integration (21) and the energy balance (23) produce the same damping coefficients illustrating the accuracy of the calculations. Note that the pressure integration of $b_{11}$ for the solid geometry in Fig. 3(a) is not accurate close to resonance. 


\subsection{The wave exciting force and mo- ment}

The wave exciting force and moment are obtained by $F_{j}^{e x}=\operatorname{Re}\left(\rho g A X_{j} e^{i \omega t}\right)$ where $X_{j}=\int_{S_{B}}\left(\phi_{D}^{E}-\phi_{D}^{I}\right) n_{j} d S$ $(j=1, \ldots, 6)$. The latter expression, as also analyzed by Zhao et al. (2011a), may be rewritten by use of the body boundary conditions (19) obtaining $n_{j}=\partial \phi_{j}^{E} / \partial n+(\mathrm{i} \rho b \omega / \mu)\left(\phi_{j}^{E}-\phi_{j}^{I}\right)$ and $\phi_{D}^{E}-\phi_{D}^{I}=$ $(\mu /(\mathrm{i} \rho b \omega)) \partial \phi_{D}^{E} / \partial n=(\mu /(\mathrm{i} \rho b \omega)) \partial \phi_{D}^{I} / \partial n$. This gives

$$
\begin{aligned}
X_{j}= & \int_{S_{B}}\left(\phi_{D}^{E}-\phi_{D}^{I}\right)\left(\frac{\partial \phi_{j}^{E}}{\partial n}+(\mathrm{i} \rho b \omega / \mu)\left(\phi_{j}^{E}-\phi_{j}^{I}\right)\right) d S \\
& =\int_{S_{B}}\left(\phi_{D}^{E} \frac{\partial \phi_{j}^{E}}{\partial n}-\phi_{j}^{E} \frac{\partial \phi_{D}^{E}}{\partial n}\right) d S \\
& +\int_{S_{B}}\left(\phi_{D}^{I} \frac{\partial \phi_{j}^{I}}{\partial n}-\phi_{j}^{I} \frac{\partial \phi_{D}^{I}}{\partial n}\right) d S .
\end{aligned}
$$

The latter integral in (24) is zero. The integral $\int_{S_{B}}\left(\phi_{D}^{E} \partial \phi_{j}^{E} / \partial n-\phi_{j}^{E} \partial \phi_{D}^{E} / \partial n\right) d S$ is equal to $-\int_{S_{\infty}}\left(\phi_{D}^{E} \partial \phi_{j}^{E} / \partial n-\phi_{j}^{E} \partial \phi_{D}^{E} / \partial n\right) d S$ where the free surface boundary condition of the potentials has been used. Noting that $\phi_{j}^{E}(j=1, \ldots, 7)$ satisfy the same radiation condition at infinity we obtain

$$
X_{j}=-\int_{S_{\infty}}\left(\phi_{0} \frac{\partial \phi_{j}^{E}}{\partial n}-\phi_{j}^{E} \frac{\partial \phi_{0}}{\partial n}\right) d S
$$

The exciting force acting on the porous geometry is thus obtained by a generalized Haskind relation, formally of the same form as for a solid body, see e.g. Newman (1977, p. 303). By carrying out the integration in the far field we obtain the exciting force by the far field amplitude of the radiation potential obtained in (14), i.e., $X_{j}=H_{j}(\beta+\pi)$ ( $\beta$ the incoming wave angle).

The exciting forces $X_{1}$ and $X_{3}$ are visualized in Figs. 4(a,b), respectively. The forces are up to $X_{1,3} / R_{0}^{2} \sim 0.3$ for $\rho b \sqrt{g R_{0}} / \mu=3.13$ and approximately the half for $\rho b \sqrt{g R_{0}} / \mu=6.26$ where calculations are performed for $0<K R_{0}<2.5$. Note that $X_{1}=0$ at the resonance at $K R_{0}=\pi / 2$ corresponding to a wave length of $\lambda / R_{0}=4$. Pressure integration and the Haskind relation give the same force and is another check of the computations. Depending on the wavenumber $K R_{0}$ the exciting forces on the solid geometry are 5-20 times greater than those on a porous geometry with $\rho b \sqrt{g R_{0}} / \mu=6.26$.

In the diffraction problem the energy flux of the incoming waves contributes to a) a time averaged energy loss at the porous geometry obtained by $\overline{\int_{S_{B}} \Delta p w_{n} d S}$, and b) an energy flux due to the scattered waves which couple to the incoming waves. The time averaged energy flux of the waves evaluated at the far field becomes $\overline{\int_{S_{\infty}}-\rho\left(\partial \tilde{\phi}_{D}^{E} / \partial t\right)\left(\partial \tilde{\phi}_{D}^{E} / \partial n\right) d S}$. By carrying out the integration we obtain

$$
\begin{aligned}
0=\frac{\rho b \omega}{\mu} \int_{S_{B}}\left|\Psi_{D}\right|^{2} d S & \\
& +\frac{K}{4 \pi} \int_{0}^{2 \pi}\left|H_{7}(\theta)\right|^{2} d \theta-\frac{1}{2} \operatorname{Im}\left(H_{7}(\beta)\right) .
\end{aligned}
$$

The net far field energy flux corresponding to the negative of terms two and three in (26) is dissipated in the porous geometry at exactly the same rate, corresponding to the first term in (26), see Fig. 4(c).

\section{Mean drift force}

The expression for the mean drift force on a porous geometry is a generalization of the similar analysis for a solid geometry. This has been obtained by Zhao et al. (2011a). They also obtained calculations of the force on a truncated cylinder geometry assuming an eigenfunction expansion. Our comparison with their calculations, particularly the case with a solid geometry, which they also include in their paper, shows that there is an error in their calculations in the sense that their force is a factor of 2.5 too large. The expression of the drift force is here rederived. New calculations are presented for both porous and solid geometries. The calculcations of the solid body are compared to published calculations for the hemisphere and truncated cylinder (Grue and Biberg, 1993) as well as asymptotics in the short wave range (Molin, 1994). By dividing the results of Zhao et al.(2011a) of a factor $\pi^{2} / 4$ we obtain a correspondence between our and their results, both in the porous and solid geometry case.

Conservation of momentum of the external fluid gives

$$
\begin{aligned}
& \frac{d}{d t} \int_{V^{E}} \rho \mathbf{v}^{E} d V= \\
& -\rho \int_{S_{\infty}}\left[\left(\frac{p^{E}}{\rho}+g z\right) \mathbf{n}+\mathbf{v}^{E} \mathbf{v}^{E} \cdot \mathbf{n}\right] d S \\
& -\rho \int_{S_{B}}\left[\left(\frac{p^{E}}{\rho}+g z\right) \mathbf{n}+\mathbf{v}^{E}\left(\mathbf{v}^{E}-\mathbf{v}_{P}\right) \cdot \mathbf{n}\right] d S .
\end{aligned}
$$

In (27) $V^{E}$ denotes the fluid volume of the external fluid, bounded by $S_{\infty}$ (a geometrical surface with zero velocity), $S_{B}$, the free surface and the bottom (for $z \rightarrow$ $-\infty)$. Eq. (27) is obtained by use of the equation of motion as well as the continuity equation. There is no momentum flux through the free surface. Similarly we obtain for the internal fluid 

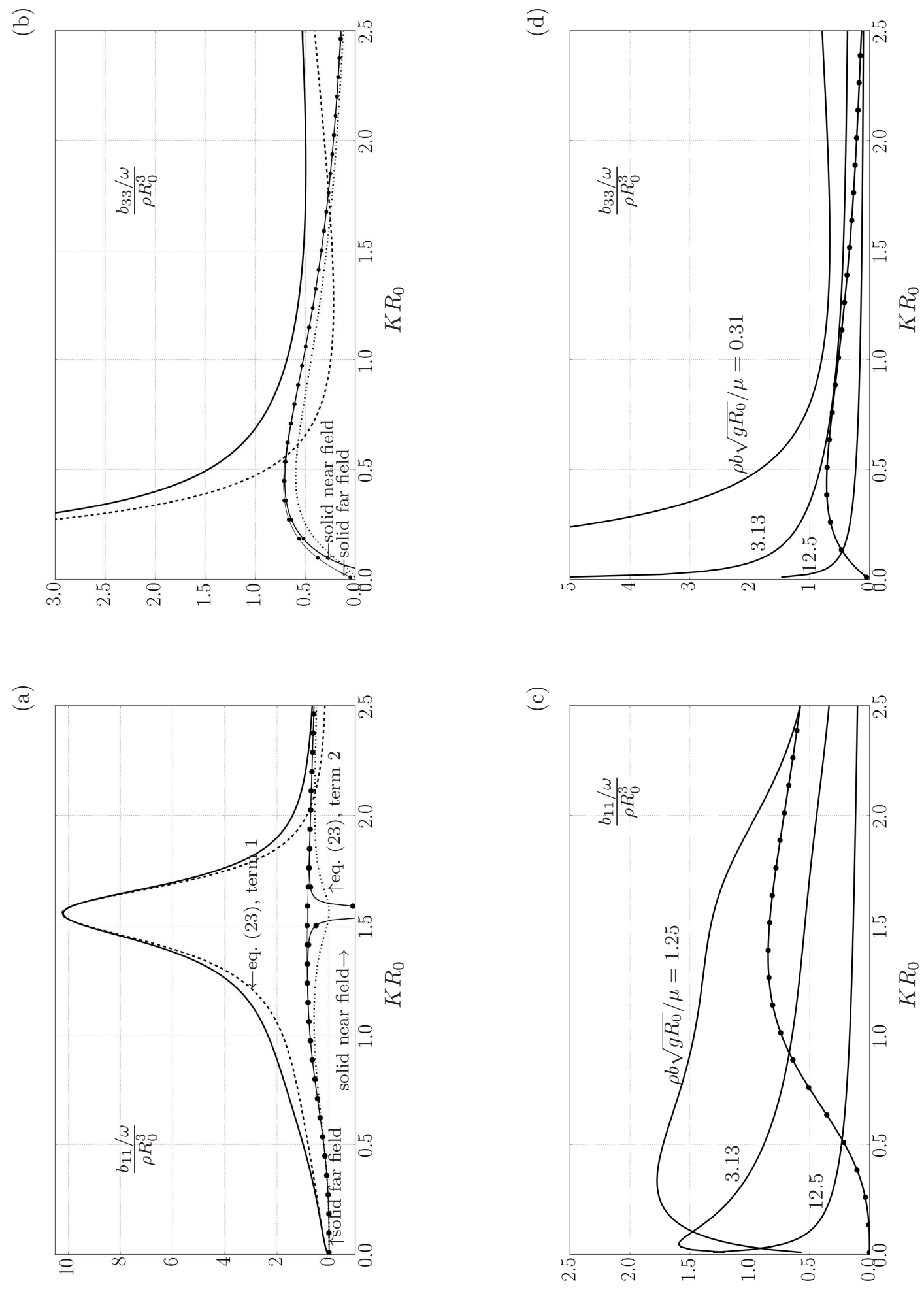

Fig. 3 Damping of hemisphere of radius $R_{0}$ at the free surface. (a) $b_{11}$ by pressure integration (21) (-), porous contribution (--), wave radiation contribution $(-\cdot-\cdot)$ and total (-) using (23) for $\rho b \sqrt{g R_{0}} / \mu=0.157$.

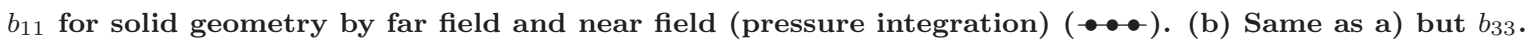

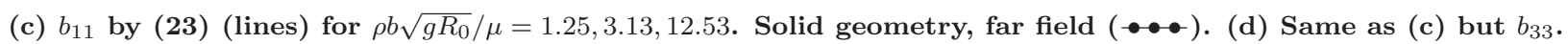




$$
\begin{aligned}
& \frac{d}{d t} \int_{V^{I}} \rho \mathbf{v}^{I} d V= \\
& \rho \int_{S_{B}}\left[\left(\frac{p^{I}}{\rho}+g z\right) \mathbf{n}+\mathbf{v}^{I}\left(\mathbf{v}^{I}-\mathbf{v}_{P}\right) \cdot \mathbf{n}\right] d S
\end{aligned}
$$

where $V^{I}$ denotes the volume at the interior of the geometry, bounded by $S_{B}$ and the free surface, and where $\mathbf{v}^{I}=\nabla \tilde{\phi}^{I}$. (The normal $\mathbf{n}$ points into $V^{I}$.) Evaluating the time average of (27-28), assuming that there is no net total mass flux, the mean drift force, $\overline{\mathbf{F}}=\overline{\int_{S_{B}}\left(p^{E}-p^{I}\right) \mathbf{n} d S}$, is obtained by

$$
\begin{aligned}
\overline{\mathbf{F}} & =-\overline{\int_{S_{\infty}}\left[\left(\frac{p^{E}}{\rho}+g z\right) \mathbf{n}+\mathbf{v}^{E} \mathbf{v}^{E} \cdot \mathbf{n}\right] d S} \\
& -\rho \overline{\int_{S_{B}}\left(\mathbf{v}^{E}-\mathbf{v}^{I}\right)\left(\mathbf{v}^{E}-\mathbf{v}_{P}\right) \cdot \mathbf{n} d S},
\end{aligned}
$$

where an overline means time average. For the horizontal force along the $x$-direction the former term in (29) attains the usual far field contribution, see e.g. Grue and Biberg (1993, eq. (48)):

$$
\begin{aligned}
\frac{\bar{F}_{x}^{(i)}}{\rho g A^{2}} & =-\frac{K}{8 \pi} \int_{0}^{2 \pi}\left|H_{7}(\theta)\right|^{2} \cos \theta d \theta \\
& -\frac{1}{2} \operatorname{Im}\left(H_{7}(\beta)\right) \cos \beta,
\end{aligned}
$$

with $H_{7}$ given by (15), where the effect of porosity appears in the boundary condition for $\partial \phi_{D}^{E, I} / \partial n$ on $S_{B}$, see (19). The second integral in (29) becomes, in the diffraction problem, with $\mathbf{v}_{P} \cdot \mathbf{n}=\operatorname{Re}\left(\mathrm{i} \omega \xi_{j} n_{j} e^{\mathrm{i} \omega t}\right)=0$,

$$
\frac{\bar{F}_{x}^{(i i)}}{\rho g A^{2}}=-\frac{1}{2 K} \operatorname{Re}\left[\int_{S_{B}}\left(\frac{\partial \phi_{D}^{E}}{\partial x}-\frac{\partial \phi_{D}^{I}}{\partial x}\right)\left(\frac{\partial \phi_{D}^{E}}{\partial n}\right)^{*} d S\right],
$$

where a star denotes complex conjugate. The total horizontal drift force becomes $\bar{F}_{x}=\bar{F}_{x}^{(i)}+\bar{F}_{x}^{(i i)}$.

Calculations illustrate the horizontal drift force on the fixed hemisphere (in the diffraction problem) where the contribution from the porous term (31) is seen to be the larger, while the contribution (30) from the scattered waves in the far field is small to moderate, where the porosity factor is $\rho b \sqrt{g R_{0}} / \mu=2.19$ in Fig. 5(a). Results for a truncated cylinder of draught $d / R_{0}=1$ are included as well. The considerable contribution to the drift force in the long wave regime for the porous geometry differs fundamentally from that on the solid body. This is further illustrated in Fig. 5 (b) for $\rho b \sqrt{g R_{0}} / \mu$ in the range $\sim 0.31-6.26$. The drift force on the porous geometry grows almost linearly with the wavenumber when $K R_{0}<<1$ and means that a body of even moderate radius is exposed to a severe drift force in the long wave regime. This effect of the porous geometry is also observed in the calculations by Zhao et al. (2011a). Note also that the viscous effect is known to enhance the mean drift force on semi-submersibles in the long wave regime, where the contributions from a pure potential formulation underpredict the forces. This is well known to specialists in ocean engineering (Nestegård, 2017, personal communication).

Fig. 5(c) obtains the drift force as function of the parameter $G_{0}=\rho b \omega /(\mu K)$ for wavenumbers in the range $K R_{0} \sim 0.25-2.5$ where the value of the ${ }^{\prime} b^{\prime}=B_{0^{-}}$ parameter of 8.5 in Zhao et al. (2011a) corresponds to $G_{0}=B_{0} /(2 \pi)=1.35$ in our notation, see (17). Note from Fig. 5(c) (for the hemisphere), for $G_{0}=10$ ( $B_{0}=$ $20 \pi), \bar{F}_{x} \simeq 0.1 \rho g A^{2} R_{0}$ for $K R_{0}=0.25,0.45,1.01$ and $\bar{F}_{x} \simeq 0.07 \rho g A^{2} R_{0}$ for $K R_{0}=2.5$. For illustrative purposes we estimate from table 1 an opening rate of the geometry of $\tau \sim 0.14$ for $G_{0}=1.35\left(B_{0}=8.5\right)$ and of $\tau \sim 0.43$ for $G_{0}=10\left(B_{0}=20 \pi\right)$, both for $K A=0.05$.

Table 2 compares the present drift force calculations on the porous hemisphere and the truncated cylinder. Note that the drift force on the two different geometries are rather close. We also compare to the mean drift force obtained by Zhao et al. (2011a, Fig. 5) for a truncated porous cylinder, as reproduced here in Fig. 5(d). The results of Zhao et al. are approximately a factor of 2.5 larger than our. Such a big discrepancy is unphysical. Note that it is unclear from the series representation of the diffraction wave potential in Zhao et al. (2011a, (5a)) how the flow below the truncated cylinder is represented. It is also unclear if the cylinder bottom is porous or not in Zhao et al.

In order to qualify our calculations we consider the mean drift force on the solid geometry where results presented in existing publications are summarized in table 3. It is observed that the present calculations of the drift force on the non-porous hemisphere agree with those presented in Grue and Biberg (1993, Fig. 6a). Note a small discrepancy at $K R_{0}=1.6$ due to the two different discretizations of the hemisphere. Results for the truncated cylinder with draught $d / R_{0}=1$ at infinite water depth are also included. The results by Zhao et al. (2011a, Fig. 5) for the drift force on the non-porous solid cylinder geometry are approximately a factor of 2.5 larger compared to the published calculations by Grue and Biberg (1993) as well as the present calculations. Another reference is the asymptotic result of Molin (1994, eq. (6)) for the drift force on a fixed cylinder or hemisphere of radius $R_{0}$ in the short wavelength regime $\left(K R_{0}=\infty\right)$ obtaining $\bar{F}_{x}=(2 / 3) \rho g A^{2} R_{0}$, also given in table 3 . We may speculate if Zhao et al. (2011a) have multiplied their force by $\pi^{2} / 2^{2}$. If their published results for $\bar{F}_{x}$ are 


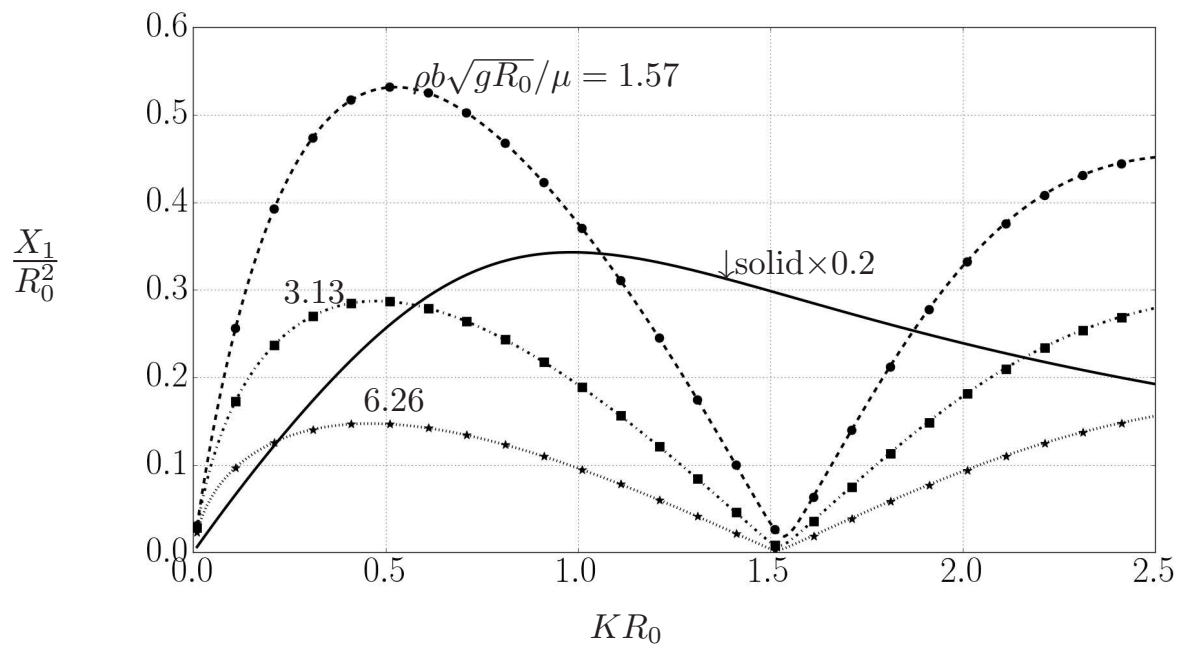

(a)

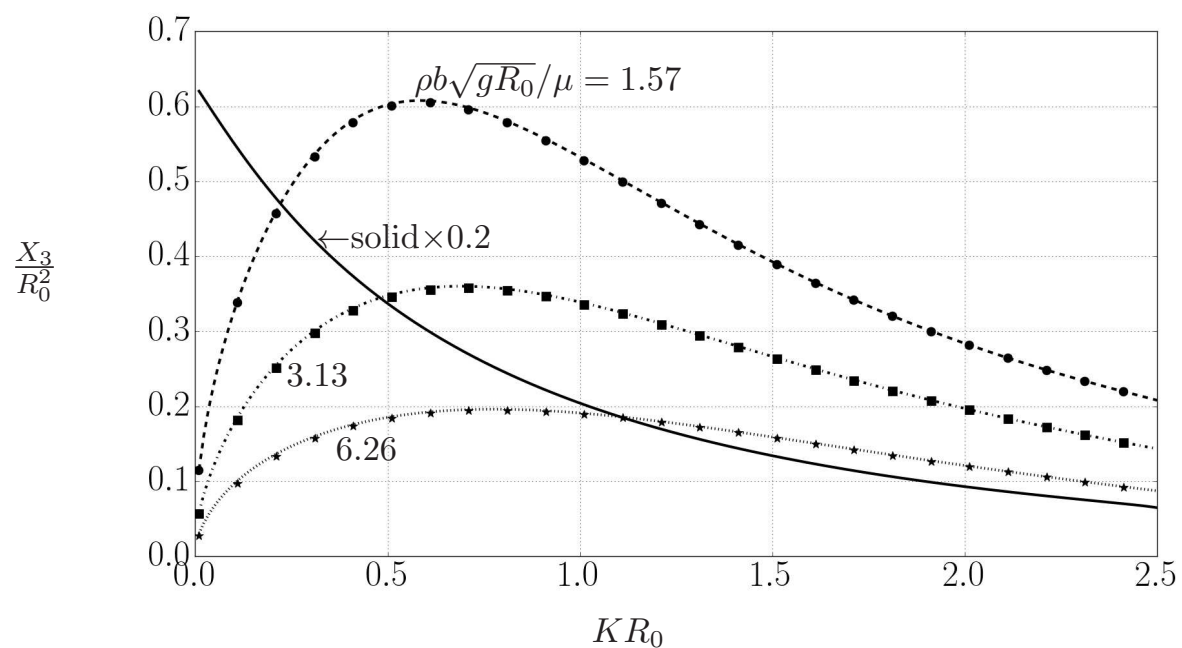

(b)

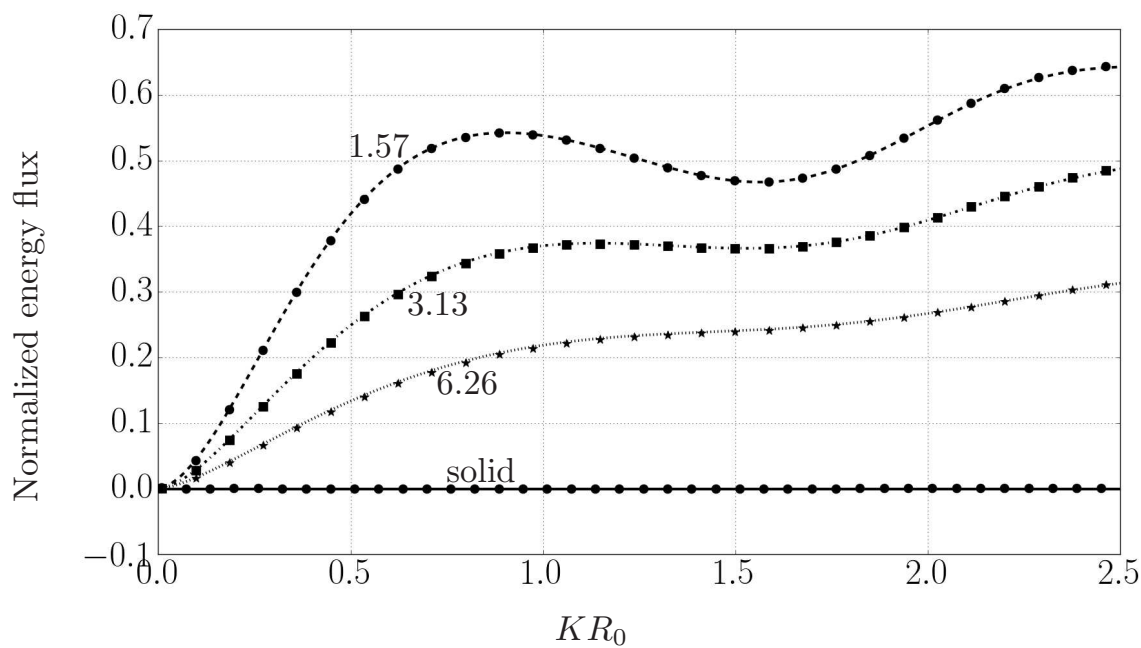

(c)

Fig. 4 Exciting force on a hemisphere by pressure integration (symbols) and Haskind relation (lines): (a) $X_{1}$ and (b) $X_{3}$. (c) The energy relation (26) with dissipation loss at $S_{B}$ (lines) and energy flux in the far field (symbols). $\rho b \sqrt{g R_{0}} / \mu=1.57,3.13,6.26$, and solid case. Wave angle $\beta=0$. 
divided by a factor of $\pi^{2} / 2^{2}$, their drift force calculations fit quite well with the present calculations for the non-porous case, see the last row in table 3 . In the case of the porous truncated cylinder case with $B_{0}=8.5$ their calculations underpredict our calculations by 20 $\%($ at $K R=1.01)$ and $30 \%($ at $K R=2.5)$, see the last row in table 2 .

Table 2 Mean drift force $\bar{F}_{x} /\left(\rho g A^{2} R_{0}\right)$ on porous fixed geometry with porosity factor ' $b{ }^{\prime}=B_{0}=$ $2 \pi G_{0}=2 \pi \rho b \omega / K \mu=8.5$ and wavenumber $K R_{0}=$ $0.25,0.45,1.01,2.50$. Hemisphere (HS) and truncated cylinder (TC) of draught $d / R_{0}=1$, both of radius $R_{0}$. $N$ number of panels. PR (present). $\mathrm{Z}$ et al. (Zhao et al. (2011, Fig. 5))

\begin{tabular}{ccccc}
\hline$K R_{0}$ & 0.25 & 0.45 & 1.01 & 2.50 \\
\hline HS, $h=\infty, N=1024, \mathrm{PR}$ & 0.23 & 0.43 & 0.43 & 0.35 \\
\hline TC, $h=\infty, N=1311, \mathrm{PR}$ & 0.25 & 0.44 & 0.43 & 0.37 \\
\hline TC, Z et al. & 0.55 & 1.02 & 0.84 & 0.63 \\
\hline TC, Z et al. $/\left(\pi^{2} / 2^{2}\right)$ & 0.22 & 0.41 & 0.34 & 0.26 \\
\hline
\end{tabular}

Table 3 Mean drift force $\bar{F}_{x} /\left(\rho g A^{2} R_{0}\right)$ on fixed solid (non-porous) hemisphere (HS) and truncated cylinder (TC) of draught $d / R_{0}=1$, both of radius $R_{0}$. $\quad N$ number of panels. $h=\infty$. PR means present. GB (Grue \& Biberg (1993, Fig. 6a)), M (asymptotic value from Molin (1994, eq. (6)), $\mathrm{Z}$ et al. (Zhao et al. (2011, Fig. 5))

\begin{tabular}{ccccccc}
\hline$K R_{0}$ & 0.25 & 0.45 & 1.01 & 1.6 & 2.50 & $\infty$ \\
\hline HS $N=1024 \mathrm{PR}$ & 0.03 & 0.13 & 0.48 & 0.53 & 0.57 & - \\
\hline$H S N=784 \mathrm{~GB}$ & 0.03 & 0.13 & 0.48 & 0.52 & - & - \\
\hline TC $N=1311 \mathrm{PR}$ & 0.03 & 0.13 & 0.58 & 0.59 & 0.63 & - \\
\hline $\mathrm{M}$ & - & - & - & - & - & 0.67 \\
\hline $\mathrm{Z}$ et al. $/\left(\pi^{2} / 2^{2}\right)$ & 0.02 & 0.13 & 0.60 & 0.61 & 0.62 & - \\
\hline
\end{tabular}

\section{Conclusion}

We perform a mathematical analysis of the linear wave diffraction-radiation problem of a porous geometry localized in the surface of a inviscid fluid. The geometry may also be submerged. A linear resistance law is assumed at the geometry where the outer and inner flows are connected. The formulation valid for geometries of general shape obtains the field variables by a set of integral equations. Calculations using a panel method obtain the added mass, damping and exciting forces as well as the mean drift force on porous geometries including a submerged sphere and a hemisphere as well as a truncated cylinder located in the free surface. The energy loss at the porous geometry and the far field energy flux are evaluated providing global checks on the computations. The generalized Haskind relations are evaluated as well, with excellent agreement to the local pressure integration of the exciting forces.

The added mass and damping of a body in unbounded fluid are function of the porosity parameter $\rho \omega b R_{0} / \mu$. In the more general case of a porous geometry interacting with a free surface suitable proxies include the wavenumber $K R_{0}=\omega^{2} R_{0} / g$ and a nondimensional porosity parameter $\rho \omega b \sqrt{g R_{0}} / \mu$. Other publications have used $G_{0}=\rho \omega b /(K \mu)$ (see Chwang, 1983; Chwang and Li, 1983) and ' $b{ }^{\prime}=B_{0}=2 \pi G_{0}=$ $2 \pi \rho \omega b /(K \mu)$ (see Zhao et al., 2011a) as dimensionless porosity parameter, in addition to the wavenumber. Experiments by Zhao et al. (2011b) provide a relation between the porosity parameter and the opening rate of the material, $\tau$, see eq. (17) in section 3. Their and our porosity parameters give an opening rate of the material of $\tau=0.12$ for $\rho \omega b \sqrt{g R_{0}} / \mu=1, K R_{0}=1$, and $\tau=0.43$ for $\rho \omega b \sqrt{g R_{0}} / \mu=10, K R_{0}=1$.

The added mass of a porous geometry located in the free surface is close to zero for a porosity parameter $\rho \omega b \sqrt{g R_{0}} / \mu>3$. The porous geometry significantly enhances the damping in the long wave regime. The exciting force $X_{1,3} / R_{0}^{2}$ is up to $\sim 0.3$ for all $K R_{0}$ and $\rho b \omega \sqrt{g R_{0}} / \mu=3.13$, and up to about the half for $\rho b \omega \sqrt{g R_{0}} / \mu=6.26$ and is a factor of 5-20 smaller than the exciting force on a solid hemisphere.

The mean horizontal drift force $\bar{F}_{x}$ on the porous geometry is significantly larger in the long wave regime compared to that on a non-porous geometry. The force $\bar{F}_{x}$ is found to grow almost linearly with the wavenumber and means that a body of even moderate radius is exposed to a severe drift force in the long wave regime. This effect of the porous geometry is also found in the calculations by Zhao et al. (2011a). Note that a viscous effect is known to enhance the mean drift force on semi-submersibles in the long wave regime, where the contributions from a pure potential formulation underpredict the forces, according to specialists in ocean engineering (Nestegård, 2017, personal communication).

The present evaluation of the mean drift force for both porous and non-porous geometries, with comparison to existing publications regarding the mean 
drift force on a solid hemisphere and truncated vertical cylinder, shows that the numerical values of $\bar{F}_{x}$ obtained by Zhao et al. (2011a) are a factor $\pi^{2} / 2^{2}$ too large, both for the porous and non-porous case. Dividing by this factor, the results by Zhao et al. still underpredict the drift force on a porous body by a factor of 20-30 per cent for $K R_{0}>1$. The present analysis and calculations provide important references to a similar wave analysis assuming a quadratic resistance law at the porous geometry.

\section{Acknowledgement}

The wave radiation-diffraction program WAMIT (version 5.3) was provided by Massachusetts Institute of Technology and Det Norske Veritas (now DNV-GL) through a mutual agreement with University of Oslo in 1994.

\section{References}

An S, Faltinsen OM, 2012. Linear free-surface effects on a horizontally submerged and perforated 2D thin plate in finite and infinite water depths. Appl. Ocean Res. 37, 220-234.

DOI: https://doi.org/10.1016/j.apor.2012.04.006

Behera H, Koley S, Sahoo, T 2015. Wave transmission by partial porous structures in two-layer fluid. Engng. An. with Bound. Elements 58, 58-78. https://doi.org/10.1016/j.enganabound.2015.03.010

Chwang AT, 1983. A porous-wavemaker theory. J. Fluid Mech. 132, 395-406. DOI: https://doi.org/10.1017/S0022112083001676

Chwang AT, Wu J, 1994. Wave scattering by submerged porous disk. J. Eng. Mech. 120, 2575-2587. https://doi.org/10.1061/(ASCE)0733-9399(1994) 120:12(2575)

Chwang AT, Chan AT, 1998. Interaction between porous media and wave motion. Annu. Rev. Fluid Mech. 30, 53-84. https://doi.org/10.1146/annurev.fluid.30.1.53

Faltinsen OM, 1990. Wave loads on offshore structures. Annu. Rev. Fluid Mech. 22, 35-56.

Finne S, Grue J, 1997 On the complete radiationdiffraction problem and wave-drift damping marine bodies in the yaw mode of motion. J. Fluid Mech. 357, 289-320. DOI: https://doi.org/10.1017/S0022112097008240

Grue J, Biberg D, 1993. Wave forces on marine structures with small speed in water of restricted depth. Appl. Ocean Res. 15, 121-135. https://doi.org/10.1016/0141-1187(93)90036-W

Grue J, Palm E, 1993. The mean drift force and yaw moment on marine structures in waves and current. J. Fluid Mech. 250, 121-142.

DOI: https://doi.org/10.1017/S0022112093001405

Grue J, Palm E, 1996. Wave drift damping of

floating bodies in slow yaw-motion.

J. Fluid Mech. 319, 323-352.

DOI: https://doi.org/10.1017/S0022112096007367

Huang Z, Li Y, Liu Y, 2011. Hydraulic performance and wave loadings of perforated/slotted coastal structures: A review. Ocean Engng. 38, 10311053. https://doi.org/10.1016/j.oceaneng.2011.03.002

Jarlan GE, 1961. A perforated vertical wall breakwater. Dock and Harb. Auth. XII 486, 394-398.

Koley S, Kaligatla RB, Sahoo T, 2015a. Oblique wave scattering by a vertical flexible porous plate.

Stud. Appl. Math. 135, 134.

DOI: $10.1111 /$ sapm. 12076

Koley S, Behera H, Sahoo T, 2015b. Oblique wave trapping by porous structures near a wall.

J. Engng. Mech. 141(3), 1-15. https://doi.org/10.1061/(ASCE)EM.19437889.0000843

Laws EM, Livsey JL, 1978. Flow through screens. Annu. Rev. Fluid Mech. 10, 247-266.

Liu Y, Li HJ, 2013. Wave reflection and transmission by porous breakwaters: a new analytical solution. Coast. Engng. 78, 4652. https://doi.org/10.1016/j.coastaleng.2013.04.003

Molin B, 1994. Second-order hydrodynamics applied to moored structures - A state-of-the-art survey. Ship Technology Res. 41, 59-84.

Molin B, 2001. On the added mass and damping of periodic arrays or partially porous disks. J. Fluids and Struct. 15, 275-290. doi:10.1006/jfs.2000.0338

Molin B, 2011. Hydrodynamic modeling of perforated structures. Appl. Ocean Res. 33, 1-11. DOI: https://doi.org/10.1016/j.apor.2010.11.003

Molin B, Remy F, 2013. Experimental and numerical study of the sloshing motion in a rectangular tank with a perforated screen.

J. Fluids and Struct. 43, 463-480. http://dx.doi.org/10.1016/j.jfluidstructs.2013.10.00

Newman JN, 1977. Marine hydrodynamics. MIT Press, 402 pp.

Newman JN, 2014. Cloaking a circular cylinder in water waves. Eur. J. Mech. B/Fluids 47, 145-150. http://dx.doi.org/10.1016/j.euromechflu.2013.11.005

Nossen J, Grue J, Palm E, 1991. Wave forces on three-dimensional floating bodies with small forward speed. J. Fluid Mech. 227, 135-160. DOI: https://doi.org/10.1017/S002211209100006X

Taylor GI, 1956. Fluid flow in regions bounded by porous surfaces. Proc. Roy Soc. Lond. A 234(1199), 456-475. DOI: $10.1098 /$ rspa. 1956.0050 

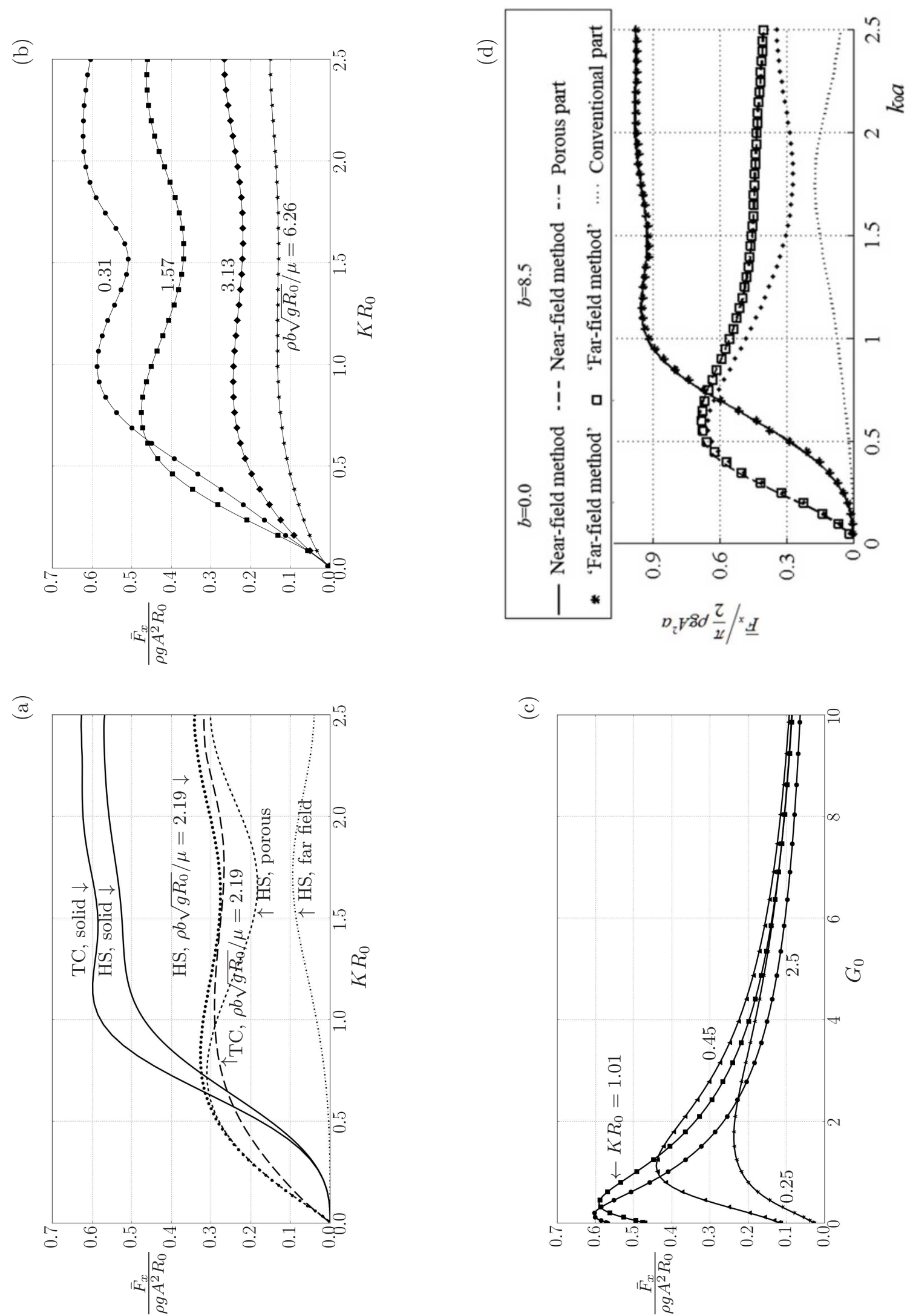

Fig. 5 Mean drift force. Fixed hemisphere (HS) and truncated cylinder (TC). (a) $\bar{F}_{x}$ vs. $K R_{0}$, for $\rho b \sqrt{g R_{0}} / \mu=2.19$, for HS (thick dots) and TC (long dashes), porous part for HS $\bar{F}_{x}^{i i}$ (thin dashed) and far field for HS $\bar{F}_{x}^{i}$ (b) (thin dash-dot). Solid HS and TC (-). $\bar{F}_{x}$ vs. $K R_{0}$ for $\rho b \sqrt{g R_{0}} / \mu=0.31,1.57,3.13,6.26$. (c) $\bar{F}_{x}$ vs. $G_{0}=$ $\rho b \omega /(K \mu)$ for $K R_{0}=0.25,0.45,1.01,2.50$. (d) Zhao et al. (2011a, Fig. 5) for $\bar{F}_{x}$ for ${ }^{\prime} b^{\prime}=B_{0}=8.5$ (porous) and ${ }^{\prime} b^{\prime}=B_{0}=0$ (solid) TC, draught $d / a=1$, water depth $h / a=3$, cylinder radius $a$. 
Willams AN, Li W, Wang K-H, 2000. Water wave interaction with a floating porous cylinder. Ocean Engng. 27, 1-28. https://doi.org/10.1016/S0029-8018(98)00078-X

$\mathrm{Yu}$ X, 1995. Diffraction of water waves by porous breakwaters. J. Waterway, Port, Coastal, Ocean Engng. 121, 275282. https://doi.org/10.1061/(ASCE)0733-950X (1995)121:6(275)

Zhao F, Kinoshita T, Bao W, Wan R, Liang Z, Huang L, 2011a. Hydrodynamics identities and wave-drift force of a porous body.

Appl. Ocean Res. 33, 169-177. DOI: https://doi.org/10.1016/j.apor.2011.04.001

Zhao F, Bao W, Kinoshita T, Itakura H, 2011b. Theoretical and experimental study of a porous cylinder floating in waves. J. Offsh. Mech. Arctc. Engng. 133/011301-1-10. doi:10.1115/1.4001435 